\title{
Thrombophilie häufiger als gedacht
}

Lucchi G et al. Superficial vein thrombosis in non-varicose veins of the lower limbs and thrombophilia. Phlebology $2018 ; 33: 278-281$. doi:10.1177/0268355517690643

Ein Thrombophilie-Screening ist teuer, deshalb lehnen Fachgesellschaften bei oberflächlichen Venenthrombosen (SVT) einen Automatismus ab. Andererseits können bei unerkannten Fällen weitere thromboembolische Erkrankungen auftreten, mit Leid für die Erkrankten und Kosten für die Gemeinschaft. Daher ist wichtig zu wissen, wie oft bei der Risikogruppe mit SVT ohne Varizen eine Thrombophilie besteht. Die Arbeitsgruppe untersuchte 73 Patienten mit SVT der Unterschenkel auf die Prävalenz von Gerinnungsstörungsstörungen, die zu Thrombosen prädisponieren. Zu den Ausschlusskriterien gehörten u. a. Bindegewebserkrankungen, arterielle Verschlusskrankheit der unteren Extremitäten, Leberzirrhose und fortgeschrittene Niereninsuffizienz. Alle Studienteilnehmer erhielten neben dem ThrombophilieScreening weitere Blutanalysen, eine Abklärung des tiefen Venensystems und eine
Tumorsuche inklusive PSA bei den Männern und Mammografie bei den Frauen.

Mit durchschnittlich 42 Jahren waren die 44 Männer und 29 Frauen vergleichsweise jung. Die SVT bezog in 58 Fällen die V. saphena magna und in 10 Fällen die V. saphena parva ein. 14 Thrombosen betrafen Seitenäste. Eine Assoziation zu einer tiefen Beinvenenthrombose kam 9-mal vor. Bei 26 Patienten ließen sich Differenzialdiagnosen zur Thrombophilie als Ursache der SVT ermitteln (u. a. Hormontherapie, Schwangerschaft, rezente OPs, lange Flüge, Bettlägerigkeit). 60 Erkrankte erhielten ein umfassendes ThrombophilieScreening. 14 Personen wiesen keine Gerinnungsstörung auf (24\%). 46 Fälle waren positiv (76\%). Bei 5,4\% der Patienten bestätigte sich ein Malignom. Die häufigste Thrombophilie betraf den Faktor V Leiden, gefolgt von 23 MTHFR-Mutationen (38,3\%). Antiphospholipid-Antikörper kamen bei 8,3\%, Prothrombin-G20210AMutationen bei 3,3\% sowie Protein-C- und Protein-S-Defizite bei jeweils 1,6\% vor. Ein Antithrombinmangel bestand in keinem Fall. Fast jeder 3. Patient wies eine kombinierte Störung auf.

Die Autoren schlussfolgern, dass Thrombophilien nicht als Raritäten zu betrachten sind. Sie empfehlen bei Patienten mit SVT ohne Varicosis die systematische Abklärung mit dem Ausschluss anderer Auslöser und ggf. einem umfassenden Gerinnungsscreening. Die relative kleine Anzahl von Karzinomen und geringe Prävalenz von tiefen Beinvenenthrombosen begründen sie mit dem jungen Alter der Erkrankten. Einschränkend verweisen Lucchi et al. auf die geringe Fallzahl und die Notwendigkeit größerer Studien.

Dr. med. Susanne Krome, Melle 